\title{
Ensino de bem-estar e dor animal em cursos de medicina veterinária no Brasil
}

\author{
[Teaching pain and animal welfare in Veterinary Medicine courses in Brazil] \\ T.D. Borges, E.C.O. Sans, J.S. Braga, M.F. Machado, C.F.M. Molento \\ Universidade Federal do Paraná (LABEA/UFPR) - Setor de Ciências Agrárias
}

\begin{abstract}
RESUMO
Currículos de medicina veterinária devem disponibilizar ferramentas para que os futuros profissionais atendam a demanda da sociedade, que inclui preocupações diretas com os animais. O objetivo deste trabalho foi traçar um perfil do panorama geral do ensino da medicina veterinária em relação a questões de bem-estar e dor animal. O método utilizado foi análise de documentos disponíveis online e coleta de dados via aplicação de questionários para coordenadores de curso de medicina veterinária. A descrição do curso, sua grade curricular e ementário foram estudados. Observou-se que $46 \%$ das 94 instituições estudadas apresentavam a disciplina de bem-estar animal e $26 \%$ ofereciam a disciplina de etologia. Houve evidência de que há uma pronta relação com a esfera física do bem-estar animal, sendo que as outras duas esferas, comportamental e psicológica, não recebem atenção similar ao longo dos cursos. Na avaliação do ementário, o termo "bem-estar animal" é empregado com caráter difuso e o termo "dor" encontra-se presente em 54\% dos cursos estudados, relacionado principalmente a disciplinas de patologia, fisiologia, farmacologia e anestesiologia. Conclui-se que o ensino brasileiro de medicina veterinária enfatiza a esfera física do bem-estar animal, sendo importante o enriquecimento em relação às esferas comportamental e psicológica e ao ensino da dor.
\end{abstract}

Palavras-chave: bem-estar animal, currículo, dor animal, ensino, medicina veterinária

\begin{abstract}
The curricula of veterinary medicine should provide tools for future professionals to meet society demands, which include direct concerns for the animals. The overall scenario of education in veterinary medicine on issues of animal welfare and pain was studied. This study was conducted through the analysis of documents available online and via questionnaires to coordinators of veterinary medicine programs. The program description, its curriculum and course content descriptions were considered. Results show that $46 \%$ of the 94 institutions studied offer an animal welfare course and $26 \%$ offer an ethology course. We observed a direct relationship with the physical component of animal welfare; the other two components, the behavioral and psychological ones, do not receive similar attention throughout the programs. In the study of course contents of the veterinary programs, the term 'animal welfare' is used in a diffuse manner and the term 'pain' appears in 54\% of the programs studied, mainly related to disciplines covering its pathology, physiology, pharmacology and anesthesiology. We conclude that in the teaching of veterinary medicine in Brazil there is an emphasis on the physical realm of animal welfare, and that there is room for improvement in the naturalness and psychological realms and in the teaching of pain.
\end{abstract}

Keywords: animal welfare, animal pain, curricula, programs, veterinary medicine

\section{INTRODUÇÃO}

O ensino universitário no Brasil enfrenta atualmente uma proliferação do número de cursos, fenômeno que ocorre em muitas áreas da educação superior, em razão da política de aumento do número de vagas implantada pelo Ministério da Educação nos últimos anos (Braga et al., 2001). O elevado número de cursos em

Recebido em 5 de julho de 2011

Aceito em 25 de setembro de 2012

E-mail: tamaratdb@hotmail.com 
funcionamento, associado à falta de condições que possibilitem a formação de profissionais com nível mínimo de competências e habilidades necessárias para o desempenho profissional, constitui uma grave preocupação (Oliveira Filho et al., 2009). A qualidade do ensino superior tem sido debatida há tempos no Brasil, sendo necessário buscar possibilidades que propiciem soluções para o problema. Os principais elementos que conduzem à qualidade do ensino incluem o aluno, o professor e o currículo (Barros, 2005). O currículo, foco deste trabalho, não é somente um conjunto de conteúdos e uma série de orientações que se perpetuam passivamente como meio de reprodução. Ao contrário, pode ser visto como um processo de mediação entre políticas e expectativas sociais e institucionais. Assim, o currículo pode nortear a formação de diplomados aptos para a inserção em setores profissionais e para a participação no desenvolvimento da sociedade brasileira (Brasil, 1996).

A universidade, como uma instituição social, exprime a estrutura e o modo de funcionamento da sociedade como um todo (Chaui, 2003). A aspiração social atualmente tende para a demanda de mudanças significativas em relação ao trato de animais, recaindo sobre os profissionais das ciências veterinárias tal responsabilidade (Lord e Walker, 2009). De uma forma geral, todas as profissões que lidam com animais passam por esta transformação central para atender e valorizar o bem-estar animal. Neste contexto, pode haver um impasse considerando-se os currículos do curso de medicina veterinária, os quais talvez ainda não disponibilizem ferramentas adequadas para que os futuros profissionais atendam tal pleito da sociedade.

Dessa forma, no campo da medicina veterinária, pode-se perceber a necessidade de que o profissional tenha uma postura crítica em relação a assuntos ligados ao bem-estar animal (Lord e Walker, 2009). O reconhecimento da necessidade de ensino para balizar tal postura já está consolidado na literatura internacional (Hewson et al., 2005; Tadich, 2010). A Organização Mundial de Saúde (OIE), da qual o Brasil é membro, recomenda um ensino de bemestar animal obrigatório para o curso de medicina veterinária (Main, 2009; Molento e Calderón, 2009). Beaver (2005), Levine (2005) e Lord e
Walker (2009) ressaltam que as escolas de veterinária não têm enfatizado a capacitação na área de bem-estar animal de maneira suficiente. Hewson et al. (2005), em estudo com 13 universidades de diferentes regiões da Europa, América do Norte e América do Sul, destacam que a formação em bem-estar animal não segue uma mesma exigência ou padrão em todas as escolas, refletindo muitas vezes em variações de aprendizado efetivo.

Nesse sentido, conhecer a atual situação do ensino da medicina veterinária no Brasil auxiliará a tomada de decisões curriculares, na medida em que constitui a base para construção de propostas concretas acerca de quais conteúdos devem ser adicionados ou reformulados. Este trabalho teve por objetivo estudar o panorama geral do ensino da medicina veterinária no Brasil em relação à questão da relevância da dor para o bem-estar animal, avaliando grades curriculares, ementas de disciplinas e objetivos de cursos.

\section{MATERIAL E MÉTODOS}

Com o intuito de verificar quantas e quais são as instituições que oferecem o curso de medicina veterinária no Brasil, realizou-se uma pesquisa no sítio do Conselho Federal de Medicina Veterinária (CFMV, 2010). Posteriormente, efetuou-se uma busca online nos sítios das instituições cadastradas em busca da grade curricular, ementário e descrição relativa aos objetivos gerais do curso, em cada universidade.

$\mathrm{Na}$ amostra das ementas disponíveis online, realizou-se uma busca pelas palavras-chave "bem-estar animal" ou "bem estar animal" e "dor", para verificar em quais disciplinas tais termos são mencionados. A seguir, foi realizada a leitura das ementas obtidas, com foco em questões relativas à dor e ao bem-estar animal. $\mathrm{Na}$ grade curricular, foram consideradas as disciplinas de bem-estar animal e etologia, contabilizando-se quantas instituições as oferecem, se estas são obrigatórias ou optativas, suas respectivas cargas horárias e seus períodos de oferta. A descrição dos objetivos do curso foi lida na íntegra, e a abordagem proposta foi classificada de acordo com a presença de uma ou mais das seguintes quatro categorias: 1) esfera física, relacionada ao ensino da saúde e nutrição do animal; 2) esfera comportamental, relacionada à etologia animal; 3) esfera psicológica, 
relacionada às capacidades mentais dos animais; e 4) citação do termo "bem-estar animal" ou "bem estar animal", averiguando sua presença nos objetivos gerais dos cursos.

Com o intuito de aumentar a amostragem das ementas disponíveis online e não sobrepor informações, enviou-se um questionário, via correio, para as coordenações de medicina veterinária de todas as instituições cadastradas no sítio do Conselho Federal de Medicina Veterinária (CFMV, 2010). Foram efetuadas chamadas telefônicas para tais universidades, confirmando o endereço de envio e o nome do coordenador.

O questionário foi elaborado em quatro seções: 1) dados do coordenador e de sua instituição; 2) questões acerca do ensino da dor e do bemestar animal nos currículos; 3) opinião do respondente; e 4) sugestões e comentários pessoais. Na seção 2, foi perguntado se havia alguma disciplina que incluía o tema dor animal e solicitou-se aos coordenadores que listassem tais disciplinas. Estas foram, então, classificadas em nove categorias de acordo com a ênfase de ensino, a saber: I) Farmacologia; II) Anestesiologia; III) Clínica e Técnica Cirúrgica; IV) Deontologia; V) Fisiologia; VI) Introdução à Veterinária; VII) Bem-estar e Comportamento Animal; VIII) Semiologia; e IX) Não informado. Realizou-se essa classificação com o intuito de verificar qual área da medicina veterinária oferece maior atenção ao ensino da dor.

$\mathrm{Na}$ seção 3, referente às opiniões e sugestões, um dos objetivos foi verificar quão importante é o tema "A relevância da dor para o bem-estar animal" de acordo com os coordenadores do curso de medicina veterinária. Para tanto, foi solicitado a eles que pontuassem a importância do tema em uma escala de 0 a 10 . Outro objetivo dessa seção foi conhecer a opinião dos coordenadores de medicina veterinária quanto à aplicação dos conhecimentos relativos à dor animal durante a futura vida profissional do aluno. Deixou-se, ainda, um campo para que os respondentes expressassem outras opiniões pessoais.

Anexa ao questionário, foi enviada uma carta de apresentação, com informações sobre a aprovação do projeto no Comitê de Ética em
Pesquisa em Seres Humanos da UFPR, sob número de registro 0937.062.10.06 e uma breve explicação do projeto.

Os dados obtidos foram analisados por estatística descritiva em programa Excel, pacote 2007.

\section{RESULTADOS E DISCUSSÃO}

O total de instituições de ensino superior que oferecem o curso de medicina veterinária no Brasil e estão cadastradas no sítio oficial do Conselho Federal de Medicina Veterinária é de 130 entidades (CFMV, 2010). Ao ser realizada essa mesma busca no sítio do Ministério da Educação, verifica-se que esse número é de 155 instituições (Brasil, 2010). Neste trabalho foi utilizado como fonte somente o sítio do CFMV, sendo que das 130 instituições, 21 (16\%) não dispunham de informações quanto à oferta de vagas, e 109 (84\%) totalizaram 10.518 vagas anuais, sendo 2.512 vagas provenientes de universidades públicas. Tais dados demonstram que o número de vagas de universidades não pagas no Brasil representa uma pequena proporção do total.

Quanto à vinculação da instituição, 87 (67\%) são instituições particulares de ensino, e 43 (33\%) entidades públicas, das quais quatro $(3 \%)$ são municipais, 13 (10\%) estaduais e 26 (20\%) federais. Das instituições estudadas, $62 \%$ são reconhecidas pelo Ministério da Educação, as remanescentes encontram-se em processo de reconhecimento, ou com a validade do reconhecimento vencida, ou ainda não apresentaram informações (CFMV, 2010).

Considerando-se os resultados obtidos online, observou-se que, do total de instituições de ensino superior que oferecem o curso de medicina veterinária no Brasil, 94 (72\%) disponibilizaram grade curricular online, possibilitando a avaliação da presença das disciplinas de bem-estar animal e etologia. Levando-se em conta somente as 94 instituições que disponibilizam sua grade curricular online, pode-se observar que $43(46 \%)$ instituições apresentavam a disciplina de bem-estar animal e $25(26 \%)$ ofereciam a disciplina de etologia. A disciplina de bem-estar animal apresentou em 23 (25\%) instituições caráter obrigatório, em sete (7\%) das instituições a disciplina era optativa, e $13(14 \%)$ instituições não apresentavam 
informações (Tab. 1). A tendência de aumento do número de instituições que ofertam a disciplina de bem-estar animal no Brasil está descrita na literatura (Quadros e Molento, 2008; Molento e Calderón, 2009) e provavelmente está relacionada à compreensão de que, ao inserirem a disciplina de bem-estar animal em seus currículos, as universidades aumentam a adequação dos seus egressos ao mercado de trabalho atual e contribuem para um avanço na ética da relação ser humano-animal. A oferta da disciplina de bem-estar animal concentra-se entre o primeiro e o quinto (19\%) períodos. A carga horária disponível para a disciplina de bem-estar animal encontra-se majoritariamente entre 30 e 40 horas semestrais (16\%). Houve uma ampla dispersão de período de oferta e carga horária da disciplina ofertada entre as instituições estudadas.

A disciplina de etologia (Tab. 1) apresenta majoritariamente caráter obrigatório com 13 (14\%) ocorrências, sendo que disciplinas optativas aparecem em nove (10\%) ocorrências e em duas (2\%) não houve informações. Tal disciplina se concentra entre o primeiro e o quinto períodos do curso (13\%) e apresenta carga horária entre 40 e 60 horas semestrais (10\%). Assim, a disciplina de etologia é menos frequente que a de bem-estar animal, mas é ofertada com maior carga horária quando presente. As disciplinas de bem-estar animal e etologia encontram-se, de uma forma geral, em um percentual baixo nas grades curriculares dos cursos de medicina veterinária brasileira.

Tabela 1. Oferta das disciplinas de bem-estar animal e etologia, conforme consulta às grades curriculares disponíveis nos sítios institucionais de 94 cursos de medicina veterinária do Brasil cadastrados no Conselho Federal de Medicina Veterinária, fevereiro a março - 2010.

\begin{tabular}{|c|c|c|c|c|c|c|c|c|}
\hline \multirow{2}{*}{ Região } & \multicolumn{4}{|c|}{ Bem-estar animal } & \multicolumn{4}{|c|}{ Etologia } \\
\hline & Total & Obr. & Opt. & N.I. & Total & Obr. & Opt. & NI \\
\hline Norte & 2 & 2 & 0 & 1 & 0 & 1 & 0 & 0 \\
\hline Nordeste & 6 & 1 & 2 & 2 & 3 & 1 & 1 & 0 \\
\hline $\begin{array}{l}\text { Centro- } \\
\text { Oeste }\end{array}$ & 7 & 3 & 2 & 4 & 7 & 2 & 3 & 1 \\
\hline Sudeste & 15 & 9 & 1 & 4 & 8 & 3 & 4 & 1 \\
\hline Sul & 13 & 8 & 2 & 2 & 6 & 6 & 1 & 0 \\
\hline Total & 43 & 23 & 7 & 13 & 24 & 13 & 9 & 2 \\
\hline
\end{tabular}

Obr.= Obrigatória / Opt. = Optativa / NI = Não informado.

Quanto ao ementário, verificou-se que somente $22(17 \%)$ das instituições estudadas apresentavam ementas online; desta forma, os resultados obtidos são preliminares, oriundos de um percentual relativamente pequeno do total de instituições existentes. O método utilizado sugere que a amostra, embora pequena, não tenha sido tendenciosa quanto aos objetivos do trabalho.

Considerando-se as 22 instituições estudadas, a busca pelas palavras-chave "bem-estar animal" ou "bem estar animal" nas ementas disponíveis revelou que estas aparecem em 14 (67\%) das instituições. As disciplinas que abordavam esse tema variaram desde aquelas voltadas à ciência do bem-estar animal em si até aquelas nas quais bem-estar animal não constituía o tema principal (Tab. 2), o que aponta para um uso transversal do termo nos ementários brasileiros. Entre as disciplinas denominadas bem-estar animal, destaca-se o uso das palavras: bioética, etologia e comportamento animal como parte da descrição das ementas, mostrando uma tendência de associação destas com o ensino de bem-estar. Tal achado está de acordo com Gonyou (1994), que afirma existir forte relação entre os termos bemestar animal e etologia. O resultado também é coerente com Fraser e Weary (2004), que descrevem a íntima relação entre questões científicas de bem-estar animal e ética animal. Destaca-se, ainda, o fato de que em sete $(31 \%)$ das instituições o termo bem-estar animal não é mencionado em nenhuma das ementas.

A palavra-chave "dor" foi encontrada em 12 (54\%) ementas, podendo esta aparecer mais 
de uma vez no ementário. Verificou-se que $10(83 \%)$ das ocorrências estão relacionadas a aspectos fisiológicos, farmacológicos, patológicos e anestésicos da dor e em cinco (42\%) transparece a possibilidade de uma abordagem de aspectos adicionais da dor, nos casos de uma disciplina de Clínica de Equinos, uma de Prática de Atividades Interdepartamentais de Apoio de Diagnóstico, duas de Bem-estar Animal e uma de Bioética e Bem-estar Animal. Tais resultados sugerem uma maior possibilidade de abordagem de aspectos relacionados à relevância da dor para o bem-estar animal nas últimas quatro disciplinas mencionadas. Outro dado relevante é que, entre as 22 ementas analisadas, 10 (45\%) não mencionam o termo "dor". Desta forma, percebese que o ensino da dor ainda é limitado e que, na maioria das vezes, restringe-se aos aspectos fisiológicos, patológicos, farmacológicos e anestésicos. Os resultados sugerem uma possibilidade de aprimoramento quanto à formação dos médicos veterinários em relação à relevância da dor para o bem-estar animal, de maneira a promover reflexão e busca de melhorias pelos futuros profissionais.

Tabela 2. Características do ensino de bem-estar animal (BEA) e dor animal nos cursos brasileiros de medicina veterinária que disponibilizam suas ementas online, correspondendo a $17 \%$ do total de cursos cadastrados no CFMV, fevereiro a março - 2010

\begin{tabular}{lccccc} 
Região & $\begin{array}{c}\text { Total de } \\
\text { instituições } \\
\text { com ementas } \\
\text { online }\end{array}$ & $\begin{array}{c}\text { Total de } \\
\text { instituições } \\
\text { com } \\
\text { disciplinas }_{\text {de BEA }^{1}}\end{array}$ & $\begin{array}{c}\text { Total de } \\
\text { instituições com } \\
\text { outras } \\
\text { disciplinas que } \\
\text { abordam o tema } \\
\text { BEA }^{1}\end{array}$ & $\begin{array}{c}\text { Total de instituições } \\
\text { com disciplinas de } \\
\text { fisiologia, patologia } \\
\text { e farmacologia que } \\
\text { abordam o tema } \\
\text { dor }^{1}\end{array}$ & $\begin{array}{c}\text { Total de } \\
\text { instituições } \\
\text { com outras } \\
\text { disciplinas que } \\
\text { abordam o } \\
\text { tema dor }^{1}\end{array}$ \\
\hline Norte & 0 & 0 & 0 & 0 & 0 \\
Nordeste & 2 & 2 & 1 & 1 & 1 \\
Centro-Oeste & 6 & 3 & 5 & 2 & 1 \\
Sudeste & 8 & 1 & 7 & 3 & 2 \\
Sul & 6 & 4 & 6 & 10 & 5 \\
Total & 22 & 10 & 19 & & 2 \\
\hline
\end{tabular}

${ }^{\mathrm{T}}$ Cada instituição pode ter mais de uma disciplina.

Das 130 instituições estudadas, 118 (91\%) apresentaram a descrição dos objetivos gerais do curso disponibilizada online. Entre todas as instituições, 79 (61\%) abordaram cuidados com a saúde do animal, ou seja, a esfera física do bemestar animal, quatro (3\%) abordaram conceitos de comportamento animal, uma $(0,8 \%)$ instituição abordou o caráter psicológico dos animais e 20 (15\%) mencionaram o termo bemestar animal em seus objetivos gerais. Com tais resultados, pode-se observar que as instituições de ensino superior de medicina veterinária no Brasil enfatizam as questões relacionadas à saúde física do animal, caracterizando a abordagem de apenas uma esfera do bem-estar animal. O ensino da medicina veterinária, de uma forma geral, tem se fundamentado na saúde física do animal (Sambraus, 1998). Com essa visão, os cursos de medicina veterinária estruturam a formação profissional baseada em conteúdos aplicados à sanidade. Tal abordagem exclui fatores fundamentais do bem-estar de um animal, que consistem em suas necessidades etológicas e psicológicas, além de suas necessidades de saúde física, e que podem resultar em sofrimento caso não sejam atendidas (Alger, 2008). Segundo King (2009), currículos veterinários tradicionais, que abordarem essencialmente a saúde física do animal, não são mais suficientes; a profissão assimilou como um dever atuar em todas as esferas do bem-estar animal: física, psicológica e comportamental. Para tal, devem ser fornecidas ferramentas aos futuros profissionais (Lord e Walker, 2009).

Com relação às 20 (15\%) instituições que mencionaram o termo bem-estar animal em sua descrição do curso, somente oito (40\%) apresentaram de fato alguma disciplina com tal temática. Tais percentuais indicam que o uso do termo bem-estar animal é com frequência aplicado de forma difusa e de efetividade questionável, uma vez que não está associado de forma coerente com a oferta de ensino específico. Tal achado pode caracterizar uma utilização mais leiga do termo, de uso corrente 
com um significado que geralmente não é preciso (Broom e Molento, 2004).

Entre os resultados da avaliação via correio, foram obtidas respostas de 28 instituições de um total de 130, sendo que somente dois questionários enviados voltaram por motivo de mudança de endereço. A região Sudeste apresentou maior participação, com 15 (53\%). Quanto à vinculação, verificou-se que 15 (53\%) das instituições respondentes são instituições públicas de ensino superior, sendo 10 (36\%) federais e cinco (17\%) estaduais.

Deste total de 28 instituições, observou-se que o perfil dos coordenadores respondentes é de 18
$(64 \%)$ do sexo masculino, $10(36 \%)$ com idade entre 30 e 35 anos, 13 (46\%) estão no cargo de coordenador há menos de um ano e 26 (92\%) são formados em medicina veterinária, sendo que, dos outros respondentes, um (4\%) não informou sua formação e um (4\%) é formado em agronomia.

Quando perguntado se havia alguma disciplina que abordava o tema dor animal, obteve-se resposta sim de 27 (96\%) coordenadores. Os resultados referentes ao ano de oferta da disciplina, à obrigatoriedade, à carga horária da disciplina e à carga horária dedicada à dor estão descritos na Tab. 3.

Tabela 3. Distribuição das disciplinas que abordam dor, de acordo com respostas de 28 coordenadores de curso de medicina veterinária no Brasil, abril a agosto - 2010

\begin{tabular}{|c|c|c|c|c|c|}
\hline $\begin{array}{l}\text { Disciplinas que } \\
\text { abordam dor } \\
\text { animal }\end{array}$ & $\begin{array}{l}\text { Total de } \\
\text { disciplinas } \\
\text { listadas }\end{array}$ & $\begin{array}{c}\text { Ano da } \\
\text { oferta da } \\
\text { disciplina }\end{array}$ & $\begin{array}{c}\text { Disciplina } \\
\text { obrigatória } \\
\text { (\%) }\end{array}$ & $\begin{array}{l}\text { Faixa de carga } \\
\text { horária de maior } \\
\text { frequência }(\mathrm{h})\end{array}$ & $\begin{array}{c}\text { Carga } \\
\text { horária } \\
\text { dedicada à } \\
\text { dor }(\%)\end{array}$ \\
\hline Farmacologia & 20 & $2^{\circ}$ & 100 & 30 e 60 & 16 \\
\hline Anestesiologia & $23 *$ & $3^{\circ}$ & 100 & 61 e 90 & 17 \\
\hline $\begin{array}{l}\text { Clínica e Técnica } \\
\text { Cirúrgica }\end{array}$ & $15^{*}$ & $4^{\circ}$ & 100 & 61 e 90 & 16 \\
\hline Deontologia & 2 & $1^{\circ}$ e $3^{\circ}$ & 100 & 30 e 90 & 8 \\
\hline Fisiologia & 9 & $2^{\circ}$ & 100 & 30 e 150 & 15 \\
\hline $\begin{array}{l}\text { Introdução à } \\
\text { Veterinária } \\
\text { Bem-estar e }\end{array}$ & 4 & $1^{\circ}$ & 75 & 30 e 60 & 17 \\
\hline $\begin{array}{l}\text { Comportamento } \\
\text { Animal }\end{array}$ & 8 & $1^{\circ}$ & 50 & 30 e 60 & 16 \\
\hline Semiologia & 4 & $3^{\circ}$ & 100 & 61 e 90 & 17 \\
\hline Não informado & 1 & - & - & - & - \\
\hline Total & 86 & & & & \\
\hline
\end{tabular}

O ensino específico de dor utiliza, em média, somente $15,2 \%$ da carga horária total das disciplinas que estão relacionadas à dor animal. Main (2010) afirma que os currículos do curso de medicina veterinária encontram-se atualmente sob pressão contínua, de políticos, das expectativas dos estudantes e das exigências comerciais, para a inclusão de questões de bemestar animal. Entre os assuntos a serem incluídos, a preocupação com o ensino da dor animal desponta como muito relevante. A tendência provável, dessa forma, é que essa porcentagem aumente no decorrer dos anos também no Brasil.
Em relação às disciplinas que abordam o tema dor, 22 (25\%) daquelas listadas pelos coordenadores apresentavam em suas ementas o assunto de diagnóstico da dor, e 15 (17\%) não apresentavam em suas ementas nenhum aspecto referente à geração de dor animal pelo ser humano. Em contrapartida, segundo os coordenadores, as outras $13(15 \%)$ disciplinas que continham em suas ementas uma abordagem quanto à geração de dor animal pelo ser humano eram, em sua maioria, seis $(46 \%)$ disciplinas de comportamento e bem-estar animal.

Verificou-se ainda, por meio das respostas dos coordenadores, que a disciplina de bem-estar 
animal é ofertada em 18 (64\%) das instituições, sendo que destas, $11(39 \%)$ apresentavam caráter obrigatório, oito $(29 \%)$ eram ofertadas no primeiro ano e $16(57 \%)$ apresentavam carga horária entre 30 e 60 horas. $\mathrm{O}$ fato de um maior percentual de cursos com a oferta de disciplina de bem-estar animal aparecer nas respostas dos coordenadores do que na avaliação das ementas online pode estar relacionado a uma tendência maior de resposta ao questionário deste trabalho por parte de coordenadores de cursos de medicina veterinária que oferecem a disciplina de bem-estar animal.

Na seção de opiniões e sugestões, 17 (60\%) dos coordenadores atribuíram nota 10 (nota máxima) para o grau de importância do tema "A relevância da dor para o bem-estar animal", sendo a média das notas 9 $\pm 1,5$. Ainda, 28 (100\%) afirmaram que, durante a vida profissional, médicos veterinários aplicarão conhecimentos relativos à dor animal, mostrando que há uma preocupação com o assunto por parte dos coordenadores. Os seguintes comentários pessoais foram selecionados, a fim de demonstrar tal preocupação:

(1) "A dor é subestimada e como tal subcontrolada.",

(2) "Acredito que não podemos mais exercer nossa profissão médico-veterinária sem saber sobre bem-estar animal.";

(3) "Acho muito importante abordar o assunto dor de forma mais direta, para que nossos alunos tenham mais informações e maior conscientização da presença de dor em animais.".

Em síntese, foram encontrados neste trabalho resultados similares aos da América do Norte (Millman et al., 2005), nos quais o bem-estar animal como uma disciplina acadêmica é ainda um componente pequeno nos currículos. Aspectos específicos de bem-estar animal, tais como o diagnóstico e o controle da dor, são explorados de maneira esporádica em disciplinas individuais dentro da medicina veterinária.

\section{CONCLUSÕES}

O planejamento do ensino da dor e do bem-estar animal nos cursos brasileiros de veterinária menciona o termo 'bem-estar animal' de uma forma transversal, empregando este em disciplinas relacionadas à ciência do bem-estar animal e em disciplinas afins de maneira difusa. Já a terminologia 'dor' é aplicada de forma restrita, estando em sua maioria relacionada aos aspectos farmacológicos, patológicos e fisiológicos da dor. Pode-se perceber a importância de aprimoramento quanto à formação dos médicos veterinários em relação à relevância da dor para o bem-estar animal.

\section{REFERÊNCIAS}

ALGER, B. Who is responsible for animal welfare? The veterinary answer. Acta Vet. Scandinavica, v.50, Suppl. 1, S11, 2008.

BARROS, G.C. Qualidade no ensino da medicina veterinária. Rev. Cons. Fed. Med. Vet., v.11, p.67-71, 2005.

BEAVER, B.V. Introduction: Animal welfare education, a Critical Time in Veterinary Medicine. $J$. Vet. Med. Educat., v.32, p.419-421, 2005.

BRAGA, M.M.; PEIXOTO, M.C.L.; BOGUTCHI, T.F. Tendências da demanda pelo ensino superior: estudo de caso da UFMG. Cad. Pesq., n.113, julho, 2001.

BRASIL. MEC - Ministério da Educação. Disponível em: < http://emec.mec.gov.br/>. Acessado em: 3 mai. 2010.

BROOM, D.M.; MOLENTO, C.F.M. Bem-estar animal: conceitos e questões relacionadas - Revisão. Arch. Vet. Sci., v.9, p.1-11, 2004.

CFMV - Conselho Federal de Medicina Veterinária. Disponível em: http://www.cfmv.org.br/portal/index.php. Acessado em: 5 abr. 2010.

CHAUI, M.A. Universidade pública sobre nova perspectiva. Revista Brasileira de Educação, Rio de Janeiro: Editora Autores Associados, n.24, 2003.p.515. Disponível em: <http:www.scielo.br/scielo.php? script=sci_arttext\&pid=S1413-

$24782003000300002 \&$ Ing=em\&nrm=isso $>$. Acessado em: 21 mar. 2010. 
FRASER, D.; WEARY, D.M. Quality of Life for Farm Animals: Linking Science, Ethics, and Animal Welfare. In: BENSON, G.J.; ROLLIN, B.E. The WellBeing of Farm Animals - Challenges and Solutions. Iowa: Blackwell Publishing, 1.ed, 2004. p.39-60.

GONYOU, H.W. Why the study of animal behavior is associated with the animal welfare issue. J. Anim. Sci., v.72, p.2171-2177, 1994.

HEWSON, C.J.; BARANYIOVÁ, E.; BROOM, D.M. et al. Approaches to teaching animal welfare at 13 veterinary schools wordwide. J. Vet. Med. Educat., v.32, p.422-437, 2005.

KING, L.J. One world of veterinary medicine. Rev. Scientifique et Technique, v.28, p.463-467, 2009.

LDB - Lei de Diretrizes e Bases da Educação Nacional. Lei n.9.394, de 20 de dezembro de 1996.

LEVINE, E.D.; MILLS, D.S.; HOUPT, K.A. Attitudes of veterinary students at one US college toward factors relating to farm animal welfare. J. Vet. Med. Educat., v.32, p.481-490, 2005.

LORD, L.K.; WALKER, J.B. An Approach to Teaching Animal Welfare Issues at the Ohio State University. J. Vet. Med. Educat., v.36, p.276-279, 2009.

MAIN, D.C.J. Evolution of animal-welfare education for veterinary students. J. Vet. Med. Educat., v.37, p.30-35, 2010.
MAIN, D.C.J.; APPLEBY, M.C.; WIKINS, D.B. et al. Essential veterinary education in the welfare of food production animals. Rev. Scientifique et Technique, v.28, p.611-616, 2009.

MILLMAN, S.T.; ADAMS, C.L.; TURNER, P.V. Animal Welfare training at the Ontario Veterinary College. J. Vet. Med. Educat., V.34, p.447-450, 2005.

MOLENTO, C.F.M.; CALDERÓN, N. Essential directions for teaching animal welfare in South America. Rev. Scientifique et Technique, v.28, p.617$625,2009$.

OLIVEIRA FILHO, B.D.; SANTOS, F.L.; MONDADORI, R.G. O ensino da Medicina Veterinária: realidade atual e perspectivas. Rev. Cons. Fed. Med. Vet., v.15, p.69-72, 2009.

QUADROS, J.; MOLENTO, C.F.M. Ensino de bemestar animal para médicos-veterinários no Brasil: atualização 2008. In: Congresso Brasileiro de Medicina Veterinária, 35., 2008, Gramado. Anais...Gramado: Conbravet, 2008.

SAMBRAUS, H.H. Applied Ethology - It's task and limits in veterinary practice. Appl. Anim. Behaviour Sci., v.59, p.39-48, 1998.

TADICH, N.A.; MOLENTO, C.F.M.; GALLO, C.B. Teaching animal welfare in some veterinary schools in Latin America. J. Vet. Med. Educat., v.37, p.69-73, 2010. 